Artículos de Revisión

\title{
Bibliometría o altimetría: desde las métricas tradicionales a las actuales. Revisión Bibliográfica.
}

\section{Bibliometry or altimetry: from traditional metrics to present. A review.}

Carlos Roberto Puerto Sanabria https://orcid.org/0000-0001-7296-8617 1*, Ángela Díaz Moreno https://orcid.org/0000-0002-6204-491X2**, Ólman Gradis Santos : https://orcid.org/0000-0001-7403-4156 ${ }^{2 * * *}$.

*Correspondencia a: carlos.r.puerto.s@gmail.com

\footnotetext{
${ }^{1}$ Posgrado Medicina Legal y Forense UNAH y Dirección de Medicina Forense, Tegucigalpa, ${ }^{2}$ Unidad de Investigación y Docencia, Dirección de Medicina Forense, Honduras.

*Médico residente.

**Médico en servicio social, UNAH,

$* * *$ Estudiante de
} medicina, Universidad Católica de Honduras.

Recibido: May. 2020, Aceptado: Agos. 2020

Agradecimientos: A la Dra. Mireya Matamoros, por la guía proporcionada en el diseño de este trabajo y las revisiones realizadas al mismo.

\begin{abstract}
RESUMEN
Introducción: La forma en la que se mide la difusión de la información en la comunidad científica ha sufrido cambios en la última década. Objetivo: Esta revisión tiene como objetivo contextualizar al lector sobre las diferentes formas de medir el impacto de un artículo, tomando en cuenta el "sistema de medición tradicional" y el de "métricas alternativas". Metodología de búsqueda: Se realizó una búsqueda en las bases de datos "PubMed/MEDLINE", "Dialnet", "Scielo" y "ScienceDirect", utilizando las palabras clave: Bibliometría, alt métricas, métricas alternativas y factor de impacto. Conclusiones: El "sistema de medición tradicional", basado en el factor de impacto y que se apoya esencialmente en la opinión de expertos, con el fin asegurar la calidad de las investigaciones, es una herramienta aún vigente que en conjunto con las métricas alternativas o altmétricas, favorecen la difusión del conocimiento, sin que necesariamente midan de manera precisa la calidad del artículo y su impacto.
\end{abstract}

\section{PALABRAS CLAVE}

Bibliometría, Alt métricas, Métricas alternativas, Factor de impacto, Revisión bibliográfica.

\section{CITAR COMO}

Puerto-Sanabria CR, Díaz-Moreno A, Gradis-Santos OD. Bibliometría o altmetría: desde las métricas tradicionales a las actuales. Revisión Bibliográfica. Rev. cienc. forenses Honduras. 2020; 6(2):24-30. DOI: https://doi.org /10.5377/rcfh.v6i2.10713

\section{ASPECTOS ÉTICOS}

Los autores declaran que no existe conflicto de interés en la publicación de este artículo. 


\section{ABSTRACT}

Introduction: The way in which the dissemination of information is measured in the scientific community has undergone changes in the last decade. Objetive: This review aims to contextualize the reader on the different ways of measuring the impact of an article, taking into account the "traditional measurement system" and that of "alternative metrics". Metodology: A search was carried out in the databases "Medline", "Dialnet", "Scielo" and "ScienceDirect", using the keywords: Bibliometry, alt metrics, alternative metrics and impact factor. Conclusions: The "traditional measurement system is based on the citations and the impact factor. Alternative or altmetric seek to measure the dissemination of scientific articles on blogs, web portals, social networks, or bibliographic managers. It is a mistake to try to evaluate whether traditional or alternative metrics are better. These should be considered as complementary to one of the onother.

\section{KEYWORDS}

Bibliometric, Alt metric, Alternative metrics, Impact factor, Bibliographic review.

\section{INTRODUCCIÓN}

La forma en que se mide la difusión de la información en la comunidad científica ha sufrido cambios en la última década. Medios como blogs, repositorios y redes sociales facilitan la visibilidad y extensión del contenido científico, favoreciendo el acceso a los datos virtuales del investigador. Todo esto beneficia la conexión con otros académicos y aumenta la probabilidad de contacto con inversores y/o aliados. En base a lo anterior, han surgido cambios en la aplicación de métodos y modelos estadísticos destinados a medir la difusión de material científico. Estos ya no se basan solamente en las citaciones, sino también en la actividad que la información tiene en "medios no formales" 1-4. Por tanto, surge el interés de ampliar la comprensión de las nociones de visibilidad, impacto y divulgación de trabajos académicos. Esta revisión tiene como objetivo contextualizar al lector sobre las diferentes formas de medir el impacto de un artículo, tomando en cuenta el "sistema de medición tradicional" y el sistema de "métricas alternativas"

METOdOLOGÍA DE BÚSQUEdA: Se hizo una búsqueda en las bases de datos "PubMed/MEDLINE", "Dialnet", "Scielo" y "ScienceDirect", utilizando como palabras claves: Bibliometría, altmétricas, métricas alternativas y factor de impacto. Se seleccionaron artículos que facilitaran la comprensión de conceptos y contextualización de las métricas tradicionales y alternativas.

Antecedentes y conceptos: De lo Tradicional a lo Alternativo: Lo que hoy conocemos como el "sistema de medición tradicional", de la calidad científica data desde 1955, este se basa en el factor de impacto, el cual toma forma a partir de la opinión de expertos, con el fin de asegurar la calidad de los artículos de investigación. Al ser un sistema de medición cuantitativo, permite ser exacto en cuanto al número de citas, sin embargo, no toma en consideración otros factores como el impacto social de la publicación, la calidad del artículo o la relación individual artículo-investigador ${ }^{1,5}$

En 1963 Eugene Garfield lanza el Índice de Citación Científica (Science Citation Index o SCI por sus siglas en inglés), como una base de datos documental, sentando las bases para las métricas actuales. En el año 1969, Pritchard acuña oficialmente el concepto de bibliometría definiéndola como: "La aplicación de los métodos estadísticos y matemáticos a los libros y otros medios de comunicación", con el fin de crear un lenguaje común en el sistema de citación ${ }^{3,6}$.

Desde el 2004 se correlacionó un mayor número de citaciones en artículos de repositorios con acceso libre, y por ende un mayor número de descargas. 
En 2010 Priem, Taraborelli y Groth publican el documento "AltMetrics: A Manifesto", el cual sienta las bases para las métricas alternativas a nivel del artículo. Es en 2012 que miembros académicos de múltiples disciplinas, principalmente de la Sociedad Americana de Biología Celular, toman nota de dichas deficiencias y se lleva a cabo en San Francisco la Declaración sobre Evaluación de la Investigación (Declaration on Research Assessment "DORA"), de la cual se obtienen tres recomendaciones claves ${ }^{1,3}$ : 1. La necesidad de eliminar el uso de métricas basadas en revistas, en la financiación, en los nombramientos.

2. La necesidad de evaluar la investigación por sus propios méritos, y no por la revista en que se publica.

3. La necesidad de aprovechar las oportunidades que ofrece la publicación en línea.

Los indicadores bibliométricos utilizados tradicionalmente se valen de los siguientes índices estadísticos:

1. Citación: el número de veces que un artículo o material académico está mencionado en otros artículos de investigación. Es por este motivo que se atribuye mayor calidad y prestigio a aquel recurso que cuenta con mayor cantidad de citas, ya que es prueba de la retroalimentación y control de calidad del método científico. Este concepto es esencial para comprender el resto de índices ${ }^{3,4}$.

2. Factor de impacto de una revista: Término acuñado por Garfield en el 1955; se define como la cantidad de veces que se cita a una revista en un periodo de dos o cinco años de manera consecutiva y se utiliza como factor de referencia para la evaluación y jerarquización de las publicaciones ${ }^{3-5}$.

3. Índice h: Es el conteo máximo de artículos que cuenta con igual número de citas, por ejemplo: Un índice $\mathrm{h}$ de 10 significa que 10 publicaciones del investigador tienen al menos 10 citas, y el resto cuenta con menos de 10 citas. Esto es una aproximación a la cantidad y calidad de publicaciones por cierto autor 7,8 .

4. Índice g: Se creó con la intención de mejorar el índice anterior, en el cual la variable g simboliza un conjunto de publicaciones con citas según orden decreciente de manera que las que estén agrupadas primero cuenten con más citas de forma exponencial o duplicada. Ejemplo: Un índice g de $10=g^{2}$, ya que $10^{2}=100$, lo que equivale a por lo menos 100 citaciones para esas 10 publicaciones 7,9

5. Índice e: Complemento del índice g, tiene el finde contrastar entre investigadores con índices $\mathrm{g}$ similares, pero con diferentes patrones de citación. El índice e, es la raíz cuadrada del remanente del grupo de publicaciones que no se tomó en cuenta más allá del $h$, de esta manera no se pierden referencias de publicaciones de alta calidad que no se toman en cuenta en los índices anteriores ${ }^{8}$.

6. Índice i10: Mide cuántas publicaciones del investigador tienen al menos 10 citaciones o más, por ejemplo: Un índice- i10 de 20 significa que 20 publicaciones del autor tienen al menos 10 citaciones o más ${ }^{8}$.

Sin embargo, estos métodos estadísticos no toman en cuenta factores como la calidad individual del artículo, sino que en general, el de la revista en estudio. El número total de citaciones en una revista no refleja al del total de cada uno de sus artículos contenidos, ya que se estima que generalmente solo un 20\% de los artículos, aporta el $80 \%$ de citaciones para la revista. Por otro lado, los que solo estudian el artículo individualmente, lo hacen en base a una dimensión: las citaciones en otros artículos, lo que puede no reflejar su difusión y calidad real $3,8,10$.

En consonancia a lo anterior es evidente que los métodos utilizados por los sistemas de medición del impacto de un documento son claramente insuficientes, ya que no proporcionan información 
de todas las interacciones que se producen en los nuevos contextos y medios sociales ${ }^{11,12}$.

\section{¿Qué son las métricas alternativas?}

La llegada de las redes sociales, y las bases de datos Open Access han aumentado las oportunidades de difusión del material científico. Surge el interés creciente de incluir indicadores que se acoplen a los nuevos medios de difusión. Las métricas alternativas, o altmétricas, son creadas como una propuesta complementaria al sistema métrico tradicional. "AltMetrics: A Manifesto" define a las métricas alternativas como "la creación y estudio de nuevos indicadores basados en interacciones sociales y difusión del contenido académico y científico por medio de la web". Estas buscan medir la divulgación de los artículos científicos en blogs, portales web, redes sociales, o gestores bibliográficos $4,8,13-15$.

\section{Indicadores altmétricos:}

1. En medios sociales generales y académicos: número de retuits, de me gusta, de clicks, de conversaciones, hilos o menciones.

2. Blogs científicos y enciclopedias: número de comentarios, de citas, de menciones y comentarios a la entrada de blogs, así como citas en la entrada de las enciclopedias.

3. Repositorios: consultas, vistas del artículo y descargas del mismo.

4. Gestores de referencias (CiteULike, Mendeley y Connotea): la incorporación a bibliografías y las lecturas 5,10,14,15.

Ventajas de las Métricas Alternativas: las métricas alternativas ofrecen numerosas ventajas, tanto para el investigador como para el académico. El aumento acelerado de la web como medio para diseminación de literatura médica permite cuantificar el impacto que tienen las publicaciones de manera individual $3,4,8$

Los puntos favorables para el investigador son: en contraste con el Factor de Impacto de Revista, las Altmetrías se miden en tiempo real, haciendo posible que el académico identifique artículos altamente diseminados en días posteriores a su publicación, independientemente de que el Factor de Impacto no haya sido calculado previo a los primeros dos meses de publicación en la revista o la aprobación de manuscritos ${ }^{5,13,16-18}$. Suelen ser de libre acceso pues, aunque existan múltiples herramientas pagas para cuantificar la influencia del artículo, existe una variedad gratuita. Aumenta la visibilidad que posee el investigador, proveyendo enlaces hacia el perfil de éste y forjando su reputación en el ámbito científico moderno, facilitando alianzas con instituciones académicas o inversores $5,16,19$.

Ventajas para el académico: al basarse en múltiples fuentes, provee una variedad de formatos de aprendizaje para el académico, ya sea en forma de repositorios o medios audiovisuales que se adapten a sus diferentes formas de aprendizaje. Es de fácil acceso, por lo que ciertas bases de datos ya cuentan con su AltMetric Score correspondiente para cada artículo disponible en su directorio, como es el caso de Elsevier o Mendeley. Finalmente, es más fácil de entender que los índices tradicionales 5,16,20.

Desventajas de las métricas alternativas: el número de menciones de un artículo puede no ser directamente proporcional a la relevancia académica del contenido del mismo pues son susceptibles a manipulación artificial, por ejemplo: la publicación de un caso clínico sobre una enfermedad rara puede no ser útil si se trata de incluir en una serie de casos, por lo que no será relevante académicamente. Si bien, es fácilmente entendible, esto no asegura la calidad del trabajo ya que el público general también realiza menciones y al no llegar a un consenso sobre qué indicadores medir, se vuelven subjetivas 5,20,21. 
Cuadro 1. Herramientas para la medición de métricas alternativas.

\begin{tabular}{|c|c|c|}
\hline $\begin{array}{l}\text { Altmetrics.com } \\
\frac{\text { www.almetric.com }}{(5,26-30)}\end{array}$ & $\begin{array}{l}\text { Es la plataforma de } \\
\text { Scholastics y es la } \\
\text { herramienta más } \\
\text { común de métricas } \\
\text { alternativas, que reúne } \\
\text { datos de la mayoría de } \\
\text { redes sociales y otros } \\
\text { sitos como Mendeley y } \\
\text { CiteULike. }\end{array}$ & $\begin{array}{l}\text { a. Los datos de AltMetrics se encuentran } \\
\text { disponibles directamente en el artículo o base de } \\
\text { datos científica y toma en cuenta conversaciones } \\
\text { o menciones en redes sociales y diarios } \\
\text { importantes como ser el New York Times. } \\
\text { b. El atajo, o bookmarklet, de AltMetrics permite } \\
\text { ver en tiempo real los datos del artículo sin } \\
\text { importar que el lector esté suscrito a este } \\
\text { servicio. } \\
\text { c. Ofrece muchas funciones gratuitas. } \\
\text { d. Interfaz colorida y fácil de entender para el } \\
\text { usuario. }\end{array}$ \\
\hline $\begin{array}{l}\text { PlumAnalytics } \\
\text { https://plumanalytics.com } \\
\text { /learn/about-metrics/ } \\
(5,10,28,30)\end{array}$ & $\begin{array}{l}\text { Es una herramienta } \\
\text { paga del grupo editorial } \\
\text { Elsevier. Su interfaz } \\
\text { presenta el número de } \\
\text { citas, menciones, uso, } \\
\text { capturas y difusión en } \\
\text { redes sociales a través } \\
\text { de un widget: "PlumX". }\end{array}$ & $\begin{array}{l}\text { a. Incluye fuentes más variadas, por ejemplo, } \\
\text { medios audiovisuales como You Tube. } \\
\text { b. Interfaz colorida y fácil de entender por el } \\
\text { usuario. } \\
\text { c. Agrupa información del autor/es por medio de } \\
\text { ORCID, DOI y otros formatos. } \\
\text { d. Hace uso de widgets. }\end{array}$ \\
\hline $\begin{array}{l}\text { Impactstory.org } \\
\text { https://profiles.impactstor } \\
\frac{\text { y.org/ }}{(28,31)}\end{array}$ & $\begin{array}{l}\text { Es una herramienta } \\
\text { paga basada en la web, } \\
\text { utilizada por autores de } \\
\text { contenido científico. }\end{array}$ & $\begin{array}{l}\text { a. Brinda información sobre marcadores de } \\
\text { impacto variados, como medios audiovisuales y } \\
\text { repositorios populares como Slide Share. } \\
\text { b. Le permite al autor crear su perfil de } \\
\text { investigador o importarlo desde ORCID y poder } \\
\text { compartirlo con otros académicos. } \\
\text { c. Le permite el autor descargar los marcadores } \\
\text { del artículo en hojas de Excel. }\end{array}$ \\
\hline $\begin{array}{l}\text { PLOS metrics } \\
\text { https://plos.org/publish/m } \\
\text { etrics/ }^{5,28,29,32}\end{array}$ & $\begin{array}{l}\text { Es una plataforma de Alt } \\
\text { Metrías en línea } \\
\text { disponible en PLOS.org. }\end{array}$ & $\begin{array}{l}\text { a. Interfaz amigable con el usuario. } \\
\text { b. Se enfoca más en marcadores como descargas } \\
\text { de PDF y vistas que en redes sociales. } \\
\text { c. Cuenta con muchas funciones gratuitas. }\end{array}$ \\
\hline
\end{tabular}


Una sugerencia que hace la comunidad científica es que las altmétricas se deben usar en combinación con el factor de impacto, el índice h, el número de descargas y el recuento de citas, para proporcionar una imagen más amplia del impacto del artículo, para así evitar posible sesgo y manipulacion ${ }^{22-25}$.

Herramientas para la medición de métricas alternativas.

En el Cuadro 1 se describen brevemente cuatro herramientas creadas con el fin de medir las métricas alternativas de una publicación, Almetrics.com, PlumAnalytics, Impactstory.com, PLOS metrics.

\section{CONCLUSIONES}

La transformación de la forma de medir la divulgación de los trabajos científicos debe ser proporcional a la evolución de los medios de comunicación. Bajo este enunciado surgen las métricas alternativas, sin embargo, es un error desestimar la importancia que tienen las métricas tradicionales. Las altmetrías resaltan como herramientas para medir el impacto social, mientras que las tradicionales lo hacen en la medición del impacto científico. Se ha demostrado que ninguna por si sola ha sido suficiente como factor pronóstico en el número futuro de citaciones de un artículo individual, sin embargo, esta asociación mejora al ser usadas de forma conjunta. Asimismo, es un error pensar que la forma de medir la "calidad" de la comunicación científica se logra solo a través del número de citaciones, obviando el factor de alcance. Por lo anterior, podemos concluir que es un error querer evaluar si las métricas tradicionales o las alternativas son mejores. Estas deben ser consideradas como complementarias una de la otra.

\section{REFERENCIAS BIBLIOGRÁFICAS}

1. Alonso-Arévalo J, Cordón-García JA, Maltrás Barba B. Altmetrics: medición de la influencia de los medios en el impacto social de la investigación. Sist Nac Eval Acreditación Certif Calid Educ - SINEACE [Internet]. 2016 [citado 14 de marzo de 2020]; Disponible en: http://repositorio.sineace.gob.pe/repositorio///handle /sineace/5947

2. Smith DR, Watson R. Career development tips for today's nursing academic: bibliometrics, altmetrics and social media. J Adv Nurs. 2016;72(11):2654-61.

3. Gorbea Portal S, Gorbea Portal S. Una nueva perspectiva teórica de la bibliometría basada en su dimensión histórica y sus referentes temporales. Investig Bibl. 2016;30(70):11-6.

4. Travieso Rodríguez C, Ferreira de Araújo R. Indicadores altmétricos y de citación en la producción científica en ScienceOpen: estudio descriptivo para Brasil, España y Portugal. Bibl An Investig. 2018;14(2):124-137.

5. Alonso-Arévalo J. Altmetrics: la integración del impacto científico y el impacto social de la investigación. En: Epistemología, acceso abierto e impacto de la investigación científica ISBN 978-9942-972-27-09-5. Cuenca: Universidad Católica de Cuenca (Ecuador); 2016. p. 139-163.

6. Martinovich V, Martinovich V. Indicadores de Citación y Relevancia Científica: Genealogía de una representación. Dados [Internet]. 2020 [citado 28 de septiembre de 2020];63(2). Disponible en: http://www.scielo.br/scielo.php?script=sci abstract\&pi $\mathrm{d}=\mathrm{S} 0011$

$52582020000200206 \& \operatorname{lng}=e n \& n r m=i s o \&$ tlng=es

7. Díaz I, Cortey M, Olvera À, Segalés J. Use of H-Index and Other Bibliometric Indicators to Evaluate Research Productivity Outcome on Swine Diseases. PLOS ONE. 2016;11(3):e0149690.

8. Paul B, Saha I. Research rating: some technicalities. Med J Armed Forces India [Internet]. 2018 [citado 26 de abril de 2020]. Disponible en: http://www.sciencedirect.com/science/article/pii/S037 7123718301230

9. Costas R, Bordons M. Is g-index better than h-index? An exploratory study at the individual level. Scientometrics. 2008;77(2):267-88.

10. Akers KG. Introducing altmetrics to the Journal of the Medical Library Association. I Med Libr Assoc. 2017;105(3):213-5. 
11. Alonso-Arévalo J. Altmetrics pueden no seguir siendo alternativa por mucho tiempo. Rev ORL. 2016;7(3):169-177.

12. Smith ZL, Chiang AL, Bowman D, Wallace MB. Longitudinal relationship between social media activity and article citations in the journal Gastrointestinal Endoscopy. Gastrointest Endosc. 2019;90(1):77-83.

13. Santana S. Las métricas alternativas y sus potencialidades para el profesional de la salud. Rev Médica Clínica Las Condes. 2018;29(4):484-90.

14. Maggio LA, Meyer HS, Artino AR. Beyond Citation Rates: a real-time impact analysis of health professions education research using altmetrics. Acad Med J Assoc Am Med Coll. 2017;92(10):1449-55.

15. Uribe-Tirado A, Ochoa-Gutiérrez J, Ruiz-Nuñez K, Fajardo-Bermúdez M. Visibilidad e impacto altmétrico de los investigadores de la Universidad de Antioquia: metodología aplicable a universidades. Transinformação. 2019;31.

16. Trueger NS, Thoma B, Hsu CH, Sullivan D, Peters L, Lin M. The Altmetric score: A New Measure for ArticleLevel Dissemination and Impact. Ann Emerg Med. 2015;66(5):549-53.

17. Vrabel M. Beyond the Impact Factor. Oncol Nurs Forum. 2019;46(2):143-5.

18. Thelwall M, Nevill T. Could scientists use Altmetric.com scores to predict longer term citation counts? J Informetr. 2018;12(1):237-48.

19. Crotty D. Altmetrics. Eur Heart J. 2017;38(35):26478.

20. Bean JR. Altmetrics in Scientific Research: Flash in the Pan or Transformative Innovation? World Neurosurg. 2017;104:993-5.

21. Butler JS, Kaye ID, Sebastian AS, Wagner SC, Morrissey PB, Schroeder GD, et al. The Evolution of Current Research Impact Metrics: From Bibliometrics to Altmetrics? Clin Spine Surg. 2017;30(5):226-8.

22. Taberner R. Altmetrics: beyond the impact factor. Actas Dermosifiliogr. 2018;109(2):95-7.

23. Elmore SA. The Altmetric attention score: what does it mean and why should i care? Toxicol Pathol. 2018;46(3):252-5.
24. Muñoz JM, Lorenzo JME. Citas, revistas y autores: claves para la evaluación de la producción científica en ciencias de la salud. FisioGlía Rev Divulg Fisioter. 2015;2(1):9-13.

25. Zuluaga Gaviria JD. Evaluación de la actividad científica en ciencia de la información a partir de indicadores bibliométricos y altmétricos. [Tesis]. Bogotá: Pontificia Universidad Javeriana; 2017.

26. Daly JM. Making sense of altmetrics. Equine Vet J. 2016;48(4):399-400.

27. Delgado López-Cózar E, Martín-Martín A. Thomson Reuters y las altmetrics: usage counts frente a citation counts en la Web of Science. Granada: EC3, 2015. (Working Papers; 20).

28. Wimmer EN, Rethlefsen ML, Jarvis C, Shipman JP. Understanding Research Impact: A Review of Existing and Emerging Tools for Nursing. J Prof Nurs Off J Am Assoc Coll Nurs. 2016;32(6):401-11.

29. Altmetric: sources of attention. [Internet].London: Altmetric; 2020 [citado 10 de mayo de 2020]. Disponible en: https://www.altmetric.com/about-ourdata/our-sources/

30. López-Padilla D, García-Río F, Alonso-Arroyo A, Pérez Gallán M, Puente Maestú L, Segrelles-Calvo G, et al. Altmetrics analysis of Archivos de Bronconeumología from 2014 to 2018. Arch Bronconeumol Engl Ed [Internet]. 2020 [citado 26 de abril de 2020]; Disponible en:

http://www.sciencedirect.com/science/article/pii/S15 79212920300513

31. Impactstory: descubra el impacto en línea de su investigación [Internet]. 2020 [citado 10 de mayo de 2020]. Disponible en: https://profiles.impactstory.org/about

32. Huang W, Wang $P$, Wu Q. A correlation comparison between Altmetric Attention Scores and citations for six PLOS journals. PloS One. 2018;13(4):e0194962. 